\title{
El estudio de las élites políticas gubernamentales en América Latina: Panorama, agendas de investigación y desafíos metodológicos*
}

\author{
Bastián González-Bustamante ${ }^{\dagger 1} 2$ \\ ${ }^{1}$ University of Oxford \\ ${ }^{2}$ Universidad de Santiago de Chile
}

3 de noviembre de 2020

\section{Introducción}

El estudio de los gobernantes precede por bastante a la época actual, remontándose incluso hasta los griegos clásicos. En general, la gran mayoría de los modelos históricos de organización política antes del surgimiento y consolidación del Estado moderno mostraban signos de la existencia de un grupo reducido que ejercía liderazgo o dominación sobre el resto. Quizás la única excepción fueron las sociedades tribales o acéfalas, sin embargo, en sociedades sedentarias, donde ya era posible la acumulación de excedentes económicos, se advierten procesos de estratificación social y una diferenciación en el ejercicio del poder político.

\footnotetext{
${ }^{*}$ Este artículo corresponde a una versión extendida de la publicada en la revista Iberoamericana. América Latina - España - Portugal, editada por el Ibero-Amerikanisches Institut (Berlín) en cooperación con el GIGA Institute of Latin American Studies (Hamburgo) y la editorial Iberoamericana/Vervuert (Madrid/Frankfurt am Main). La versión publicada corresponde a Olivares et al. (2020), DOI: 10.18441/ibam.20.2020.74.229-259. Agradezco el apoyo financiero de la Agencia Nacional de Investigación y Desarrollo de Chile (ANID/PFCHA/72200340).

†PRS DPhil in Politics, Department of Politics and International Relations \& St Hilda's College, University of Oxford. Profesor Instructor, Departmento de Gestión y Políticas Públicas, Facultad de Administración y Economía, Universidad de Santiago de Chile. $P$ St Hilda's College, Cowley Place, Oxford OX4 1DY, $₫$ bastian.gonzalezbustamante@politics.ox.ac.uk, 而 http://users.ox.ac.uk/ shil5311, 令 https://bgonzalezbustamante.com, ORCID iD 0000-0003-1510-6820
} 
Por ejemplo, tres milenios a. C. la unificación egipcia marcó el comienzo de una tradición dinástica fuertemente asociada con la idea de que sus gobernantes descendían de los dioses. Por otro lado, en los antecedentes de la fundación de Roma también es posible encontrar ideas similares: en la Eneida se traza la fundación de la ciudad desde Rómulo y Remo, quienes descendían de Rea Silvia y Marte, dios de la guerra. Estos son solo dos ejemplos de muchos otros posibles donde la sangre, la dinastía y la divinidad fueron elementos centrales que justificaron el ejercicio del poder político por grupos reducidos. Incluso en la democracia ateniense, cuya organización política se encontraba en las antípodas de las dinastías divinizadas, el ejercicio de la ciudadanía se restringía a las familias nobles.

Aquellos elementos fueron relevantes hasta hace pocos siglos atrás cuando los procesos revolucionarios e independentistas fueron el preámbulo al auge del Estado moderno. Esto, en palabras de Weber ${ }^{1}$, implicó una reconfiguración en las formas de dominación. Se advierte un tránsito desde formas políticas preestatales, donde la dominación era eminentemente tradicional y carismática, hacia formas de organización donde se vuelve relevante la dimensión racional-legal con ciertos elementos carismáticos asociados a la expansión de la democracia representativa como forma de gobierno durante los siglos XIX y XX.

La fascinación por entender los mecanismos que modelan el poder y a quienes lo ejercen ha perdurado. Durante el último siglo el estudio de las élites ha sido uno de los tópicos predilectos en las ciencias sociales contemporáneas, particularmente desde la irrupción de los elitistas clásicos a fines del siglo XIX. Comprender a la élite política como grupo se basa en premisas sencillas desde la teoría clásica: se trata de un conjunto minoritario de individuos que tiende a perpetuarse en el ejercicio del poder, idea que se enlaza con la conocida ley de hierro de la oligarquía de Michels. Desde entonces se ha pasado por distintas etapas en la comprensión del fenómeno elitario, plasmadas en los enfoques pluralista y unitario y la influencia del trabajo de Mills a mediados del siglo pasado, hasta el estudio de las estrategias de monopolización y reproducción de los distintos tipos y formas de capital derivados principalmente del trabajo de Pierre Bourdieu ${ }^{2}$.

En este contexto, este artículo discute brevemente algunas dificultades teóricas y caracteriza el panorama reciente, así como las principales agendas de investigación en el marco del estudio de las élites gubernamentales en América Latina. Posteriormente, se abordan algunos desafíos metodológicos y se presentan unas breves conclusiones.

\footnotetext{
${ }^{1}$ Véanse algunas obras cumbre de Weber $(1919,1922)$ para más detalles.

${ }^{2}$ Sobre este punto, aplicado con cierto énfasis en América Latina, es posible revisar algunos de los siguientes trabajos: Joignant (2011a, 2012), Rovira (2011) y González-Bustamante (2013a).
} 


\section{Dificultades teóricas}

La teoría de campos y capitales ha tenido una influencia relevante en el estudio empírico de las élites durante las últimas décadas, sin embargo, en la teorización sobre los recursos, capitales y mecanismos que operan en el campo político subyacen distintas dificultades que escapan del foco de este breve artículo. A continuación, solo se abordan algunos problemas relacionados con la delimitación del campo político para efectos del estudio de las élites gubernamentales y se esbozan ciertas complejidades asociadas a las tipologías de agentes o políticos profesionales.

Cuando el foco del estudio de las élites políticas recae en la esfera gubernamental es posible advertir un primer problema teórico. Como se mencionó previamente, responder a la pregunta quién gobierna es simple desde la teoría de las élites, por lo que la identificación de los individuos debiese ser relativamente sencilla, casi mecánica, siguiendo una estrategia de análisis posicional en desmedro de enfoques decisionales o reputacionales. Sin embargo, desde una perspectiva puramente institucional, es necesario considerar la distinción entre gobierno como estructura y gobernantes como individuos. Esto se enlaza con la complejidad para ofrecer una definición inequívoca del concepto gobierno. Siguiendo la tradición politológica clásica italiana ${ }^{3}$, por ejemplo, el término government extrañamente se utiliza en los Estados Unidos, en su lugar es más común el uso de administration, que agrupa a la presidencia y a la burocracia estatal. Por otra parte, el Reino Unido tiende a utilizar términos propios de su tradición monárquica constitucional de carácter parlamentaria como her Majesty's government, cabinet governemet o party government.

A pesar de estas diferencias, el gobierno como estructura tiende a asociarse directamente con el poder ejecutivo, cuya diferenciación con el poder legislativo depende del régimen de gobierno. El poder ejecutivo en general tiende a poseer funciones principalmente relacionadas con el resguardo del Estado de derecho, la preservación del orden público y la protección de los ciudadanos. Para esto cuenta con aparatos específicos como las fuerzas de orden y la administración pública. Esto conduce a un segundo problema teórico. Dwight Waldo en su obra The Study of Public Administration (Doubleday and Company, 1955) ya advertía el problema para definir qué es la administración pública. Una definición operacional sencilla es categorizarla como la organización que busca cumplir con los fines del gobierno, lo que entonces implica entender al gobierno como un órgano decisor y a la administración pública como un aparato que implementa dichas decisiones. Esta distinción refleja la dicotomía política-administración asociada con el

\footnotetext{
${ }^{3}$ Para más detalles se recomienda revisar Bobbio (2003), Pasquino $(2000,2011)$ y Bardi y Pasquino (1994).
} 
grado de separación y las tensiones entre las esferas política y administrativa. ¿Dónde termina la élite política y comienza el servicio civil? ¿Son los altos directivos públicos o jefes de servicios parte de la élite gubernamental? Por ejemplo, Manuel Alcántara, en su conocida obra El oficio de político (Tecnos, 2012), se plantea preguntas similares al abordar el fenómeno de la profesionalización de la política y el rol de los directivos públicos.

La dicotomía política-administración, acuñada por Woodworow Wilson en 1887, ha generado una tensión práctica y epistemológica que perdura hasta hoy entre el reclutamiento de personal con base en el mérito y los nombramientos basados en confianza política. De todas maneras, en general la literatura tiende a indicar que los nombramientos políticos y el reclutamiento por mérito coexisten en la esfera gubernamental, con límites difusos que varían y se tienden a vincular al nivel jerárquico. Mientras las posiciones más relevantes suelen ser eminentemente políticas y se privilegian los nombramientos directos, las posiciones intermedias se asocian al servicio civil ordinario o carrera funcionaria y se dotan mediante sistemas basados en mérito ${ }^{4}$. Estos sistemas dependen de cada caso y pueden estar influenciados por fenómenos como el patronazgo e incluso el clientelismo en los niveles burocráticos jerárquicamente inferiores.

En este sentido, las agendas de investigación se han enfocado, por una parte, en el estudio de presidentes, ministros, viceministros y subsecretarios. Como también en el estudio de altos directivos públicos, jefes de servicio e incluso la élite burocrática o burócratas de política pública. En este punto es posible identificar el uso de diferentes términos para referirse a estos agentes, lo que se relaciona con una ontología que apela precisamente a la dicotomía política-administración ya que, por ejemplo, en el marco de la burocracia weberiana, los funcionarios o burócratas de alto nivel fueron llamados mandarines debido a la imposibilidad de remoción y a la práctica de generar una captura burocrática sobre los políticos ${ }^{5}$. Por otro lado, el tecnócrata se relaciona con un lenguaje de recursos y capitales, en el contexto del paradigma bourdesiano, que da cuenta de individuos con elevados recursos técnicos o credenciales académicas, asociadas al capital cultural de tipo institucionalizado, y un enfoque hacia la eficiencia. En estricto rigor, el tecnócrata se encuentra en las antípodas de la figura burocrática clásica pues, como sugiere Patricio Silva en su libro In the Name of Reason (Penn State University Press, 2009), trata de eliminarlo. Por último, los conceptos alto directivo o gerente público son propios del paradigma de la Nueva Gestión Pública. Cabe destacar que los principales términos que se utilizan en la literatura especializada son tecnócrata y technopol, siendo

\footnotetext{
${ }^{4}$ Peters (1995) es un buen ejemplo de este punto.

${ }^{5}$ Para profundizar en esto véase González-Bustamante (2019b).
} 
el segundo un híbrido que entrecruza elevados recursos técnicos y capital político, tal como queda en evidencia en los trabajos de Alfredo Joignant ${ }^{6}$. Si bien esta variedad tipológica es útil teórica y descriptivamente, en algunas oportunidades puede dificultar el análisis empírico por ser categorías algo difusas y sobreponerse entre sí generando problemas de medición al trabajar con técnicas cuantitativas.

\section{Panorama reciente y agendas en América Latina}

El estudio de las élites efectivamente ha sido un objeto de estudio recurrente, casi una obsesión, para las ciencias sociales durante el siglo XX, particularmente para la ciencia política y la sociología. En América Latina, sin embargo, los quiebres democráticos a mediados de la segunda mitad del siglo pasado interrumpieron abruptamente el desarrollo científico. En este escenario, gracias al surgimiento de centros de investigación privados apoyados por la cooperación internacional e instituciones desde fuera de la región, como la Fundación Ford, fue posible la reconstitución del campo intelectual, lo que a su vez fue clave en los procesos de redemocratización ${ }^{7}$. Esto es reconocido por varios académicos. Por ejemplo, Joignant y Güell en Notables, tecnócratas y mandarines (Ediciones UDP, 2011), indican que efectivamente el estudio de las élites se revitalizó en la región en el marco de los procesos de redemocratización. Esto tiene cierta lógica ya que las agendas de investigación, como los repertorios de acción en la política contenciosa, pueden naturalmente ser limitadas y constreñirse en contextos autoritarios. Este resurgimiento en el estudio de las élites también se vio influenciado por el Proyecto de Élites Parlamentarias Latinoamericanas de la Universidad de Salamanca y el liderazgo de Manuel Alcántara ${ }^{8}$.

Desde entonces es posible apreciar la proliferación de trabajos enfocados en la profesionalización de la política, congruencia élite-ciudadanía, composición social de los círculos elitarios y circulación entre esferas y campos. Una obra interesante para comprender mejor la producción de las ciencias sociales sobre élites en Argentina, Brasil y Chile durante las últimas décadas es Las élites políticas en el Sur, compilado por Vommaro y Gené (Ediciones UNGS, 2018). En este contexto, priman enfoques más bien cualitativos y mixtos, con una fuerte presencia de etnografías, entrevistas en profundidad y análisis de trayectorias y características individuales, principalmente con técnicas univariadas. Una excepción es el análisis de congruencia o brechas que generalmente

\footnotetext{
${ }^{6}$ Véanse específicamente Joignant (2011a, 2011b).

${ }^{7}$ Para más detalles al respecto véanse Morales (2016) y Cisternas y González-Bustamante (2016).

${ }^{8}$ Se recomienda revisar algunos trabajos referentes como Alcántara $(2012,2013)$.
} 
utiliza algunos índices y modelos econométricos clásicos ${ }^{9}$. Para un debate sobre estrategias metodológicas en el marco de este enfoque el libro Como estudar elites (Editora UFPR, 2015), compilado por Perissinotto y Codato, resulta muy interesante.

Por otra parte, es posible identificar diversos estudios sobre gabinetes, rotación y reclutamiento ministerial que se vinculan con enfoques más institucionales y con los procesos de formación de coaliciones. En esta línea destacan especialmente los trabajos de Cecilia Martínez-Gallardo y Marcelo Camerlo. Buena parte de estos estudios utilizan como estrategia de estimación econométrica el análisis de supervivencia, aproximación bastante apropiada para evaluar la permanencia y rotación en gabinetes y en posiciones cuyos intervalos de tiempo son rastreables ${ }^{10}$. Recientemente se han publicado tres libros que resultan clave para esta línea. Primero, The Selection of Ministers Around the World (Routledge, 2015), editado por Dowding y Dumont, y cuyos capítulos abordan las carreras ministeriales en gabinetes parlamentarios y presidenciales en distintos países. Segundo, Las élites en las Américas: Diferentes perspectivas (Editora UFPR, 2018) editado por Codato y Espinoza y que aborda varios casos de América Latina. Por último, Government Formation and Minister Turnover in Presidential Cabinets: Comparative Analysis in the Americas (Routledge, 2018), editado por Camerlo y Martínez-Gallardo, presenta varios casos de la región y encuadra la agenda de rotación y reclutamiento en el estudio del presidencialismo de coalición en el contexto del interés por estudiar las diferencias entre las democracias presidenciales.

Finalmente, resulta relevante indicar que actualmente existen grupos y observatorios que promueven directamente la investigación sobre élites políticas y económicas en América Latina. Algunos buenos ejemplos son el Observatorio de Élites Políticas y Sociales de Brasil (coordinado por Adriano Codato), el Observatorio de las Elites Argentinas (coordinado por Paula Canelo, Ana Castellani y Mariana Heredia), la Red de Élites de América Latina (coordinada por Julián Cárdenas y Francisco Robles-Rivera) y, el recientemente creado, Grupo de Trabajo del Consejo Latinoamericano de Ciencias Sociales (CLACSO) sobre Élites Empresariales, Estado y Dominación (coordinado por Inés Nercesian, Francisco Robes-Rivera y Miguel Serna).

\footnotetext{
${ }^{9}$ Algunos trabajos en esta línea, tanto de congruencia temática como entre representantes y representados, son Alcántara y Luna (2004), Luna y Zechmeister (2005), Lupu y Warner (2017), Buquet y Selios (2017) y González-Bustamante (2019a).

${ }^{10}$ Algunos ejemplos en esta línea son Camerlo y Pérez-Liñán (2015), Martínez-Gallardo (2014), Kerby (2015), Quiroz Flores (2015) y González-Bustamante y Olivares (2015, 2016, 2018).
} 


\section{Desafíos metodológicos}

Pueden existir diversas complejidades metodológicas al estudiar la élite política gubernamental. A continuación, se abordan de forma resumida tres problemas comunes relacionados con la estrategia de identificación, la confiabilidad de la información y el análisis de vínculos con redes sociales. Posteriormente, se profundiza particularmente en el riesgo de realizar comparaciones y predicciones sesgadas.

Primero, siguiendo la clásica obra de Robert D. Putnam The Comparative Study of Political Elites (Prentice Hall, 1976), es posible identificar tres estrategias para estudiar los elencos elitarios: (a) análisis posicional; (b) análisis reputacional; y (c) análisis decisional $^{11}$. La estrategia de identificación posicional es la más sencilla ya que se basa en las posiciones formales que ocupan los individuos. Por otra parte, mientras el enfoque reputacional considera los lazos e interconexiones, el decisional busca analizar los procesos de toma de decisión. Evidentemente una estrategia posicional debería ser más sencilla, sin embargo, también tiene complejidades. Mientras que el acceso a ciertas posiciones, en particular a las de mayor jerarquía, es relativamente fácil de rastrar y operacionalizar, en general la circulación y las trayectorias de los individuos es algo más complejo de analizar adecuadamente, especialmente cuando se estudian niveles jerárquicos menores. De hecho, incluso para el nivel de subsecretarios o vicepresidentes ya es difícil verificar trayectorias. Además, las carreras políticas usualmente implican intersecciones entre distintos campos, lo que es una complejidad particular cuando se estudian las interacciones entre la élite política y económica, cuestión relevante para, por ejemplo, estudios sobre puerta giratoria o cabildeo ${ }^{12}$.

Segundo, existen problemas para obtener información confiable. Esto se debe principalmente a dos fenómenos: problemas de muestreo y deseabilidad social. Por una parte, en general el muestreo constituye un desafío relevante al momento de diseñar un proyecto de investigación cuya unidad de análisis es la élite. En general es complejo diseñar una muestra probabilística, independiente de su tipo, por lo cual intentar censar o usar muestras no probabilísticas reportando la tasa de respuesta suele ser lo más habitual. Un buen ejemplo de esto es el proyecto 1100877 del Fondo Nacional de Desarrollo Científico Tecnológico de Chile para estudiar la élite política de aquel país, liderado entre 2010 y 2012 por Alfredo Joignant, del cual han surgido varias publicaciones en los últimos años ${ }^{13}$. El proyecto inicialmente consideró una primera etapa de identifi-

\footnotetext{
${ }^{11}$ También es recomendable revisar Codato (2015).

${ }^{12}$ Algunos trabajos recientes al respecto en la región son Castellani y Dulitzky (2018), Codato et al. (2019) y Maillet et al. (2019).

${ }^{13}$ Algunas de las investigaciones que han usado este conjunto de datos son Joignant et al. (2012, 2015), Delamaza (2013), González-Bustamante (2013a, 2013b), Cuevas (2015), Cuevas et al. (2015),
} 
cación de posiciones institucionales relevantes en el campo político, tanto en el poder ejecutivo como legislativo, incluso incluyendo directivas de partidos políticos y algunos organismos autónomos. Posteriormente, se identificó un núcleo elitario con base en las posiciones más relevantes, el cual se intentó censar. La tasa de respuesta fue alta para este tipo de estudios, superando el $60 \%$, sin embargo, aun así, el proceso de revisión y validación de la muestra y la base de datos fue un trabajo complejo, que se extendió hasta septiembre de 2014, momento en el cual se realizaron los ajustes finales ${ }^{14}$. Esto es porque el rastreo de trayectorias y el multiposicionamiento de individuos hace que los procesos de revisión y validación sean muy complejos, de hecho, generalmente en este tipo de estudios es posible detectar que los individuos sobredimensionan o subdeclaran sus carreras políticas, por eso es importante una verificación minuciosa. Lo segundo ocurre particularmente por temas asociados con conflictos de interés y puerta giratoria.

Los problemas anteriores están directamente asociados con la deseabilidad social y se tienden a exacerbar cuando se recaba información delicada sobre percepciones, lo que puede generar distorsiones en estudios de congruencia ideológica o identidad partidaria. Puede ser, por ejemplo, que estudiar elencos conservadores mediante encuestas sea riesgoso. La información obtenida puede ser de muy baja calidad ya que el sesgo podría ser tremendo: de pronto políticos de partidos ultraconservadores se auto perciben como keynesianos y eso es presentado como un hallazgo relevante en una investigación, cuando en estricto rigor el resultado se encuentra absolutamente sesgado si los controles adecuados no fueron incorporados durante el trabajo de campo.

Tercero, mapear relaciones puede ser una estrategia interesante y útil. En este contexto, el análisis de redes sociales puede ser una técnica con muchas potencialidades para el estudio de las élites políticas ${ }^{15}$. Este análisis permite evaluar vínculos entre individuos desde una perspectiva relacional o con base en el supuesto de que ciertas características compartidas se cristalizan en perspectivas ideológicas e incluso ontológicas diferenciadoras entre los agentes. El problema aquí es relativamente evidente. Primero, trabajar con relaciones es bastante complejo precisamente por problemas de deseabilidad social. Segundo, detrás del supuesto de cristalización, cuando el vínculo se basa en características compartidas, subyacen conceptos como el de habitus de Bourdieu, el

González-Bustamante y Garrido-Vergara (2018) y Garrido-Vergara (2020).

${ }^{14}$ Véase Joignant y González-Bustamante (2014). También es posible encontrar detalles del procedimiento de muestreo en Joignant et al. (2015), González-Bustamante y Olivares (2015) y Maillet et al. (2016).

${ }^{15}$ Para más detalles es posible revisar Urizzi Cervi (2015) y Lorrain y White (2003). Para algunos trabajos empíricos enfocados en el caso chileno particularmente véanse Espinoza (2010), Cisternas y Vásquez (2018) y González-Bustamante y Cisternas (2016, 2020). Finalmente, para teoría clásica de redes sociales se recomienda revisar Hanneman y Riddle (2005) y Wasserman y Faust (1994). 
cual opera como una estructura estructurante generando personificaciones ontológicas que se cristalizan en redes de cooperación, sin embargo, puede ser que simplemente no exista tal vínculo o que este sea una relación conflictiva, en consecuencia, el supuesto falla ${ }^{16}$.

Por último, el estudio de las élites no se encuentra exento de la influencia de la econometría en las ciencias sociales durante las últimas décadas. En este contexto, trabajar con modelos estadísticos también ha emergido como una posibilidad atractiva para estudiar las élites políticas, sin embargo, con altos riesgos de generar comparaciones y predicciones sesgadas. Esto se puede dar cuando se estudia la élite política gubernamental. Por ejemplo, para un caso $i$ se necesita contar con una variable dependiente $y$ identificable que puede reflejar la trayectoria del caso con algún índice compuesto o indicadores más simples. En este contexto, es necesario contar con varianza en y, ya sea una variable continua o dicotómica. En otras palabras, si es binaria se necesitan casos positivos $y=1$ y negativos $y=0$. Esto es indispensable para ajustar modelos básicos como mínimos cuadrados ordinarios o logísticos.

Sin embargo, en algunas ocasiones es difícil contar con $y=0$. Por ejemplo, si se estudian nombramientos y no trayectorias o permanencia, en el poder ejecutivo no es posible contar con un conjunto de aquellos individuos que fueron considerados para la posición, pero no fueron nombrados. La excepción pueden ser ciertas posiciones de alta dirección pública ya que algunos sistemas de servicio civil generan ternas o quinas antes del nombramiento, dejando registro de aquello. En los congresistas o en otras posiciones que compiten electoralmente es más sencillo porque se puede rastrear a quienes perdieron la elección. Una alternativa es con una muestra compuesta por distintas posiciones generar comparaciones entre grupos para detectar factores de nombramiento y después calcular probabilidades, sin embargo, es poco preciso.

En este punto hay que considerar la dificultad para desarrollar una estrategia de inferencia causal efectiva. En este sentido, siguiendo los trabajos de Joshua D. Angrist, Jörn-Steffen Pischke y Kosuke Imai ${ }^{17}$, se puede considerar que el resultado de la inferencia causal es la diferencia en la variable dependiente de un caso específico $i$ cuando esta es positiva y cuando es negativa, es decir, $y_{1 i}-y_{0 i}$. En consecuencia, el efecto de una variable independiente $x$, o de un tratamiento $D$ si se utilizan métodos experimentales, sería aquella diferencia. Por ejemplo, cuánto afecta $x$ a $y$ en un monto $\kappa$, lo que se conoce como el supuesto de efecto constante. Entonces es posible asumir que $y_{1 i}=y_{0 i}+\kappa$.

\footnotetext{
${ }^{16}$ Sobre habitus véase el trabajo clásico de Bourdieu (1980).

${ }^{17}$ Para más detalles véanse Angrist y Pischke (2015) e Imai (2017).
} 
Cabe indicar que el problema fundamental de la inferencia causal es que no es posible observar los resultados contrafactuales. Por ejemplo, si $x_{i}$ o $D_{i}$ generan un cambio en $y_{i}$, para estimar con precisión el efecto es necesario considerar los dos posibles potenciales resultados de $y_{i}$, en este caso, $y_{1 i}$ e $y_{0 i}$. Sin embargo, empíricamente solo se observa uno de esos dos resultados teóricamente posibles. Entonces, si se comparan grupos con el efecto causal promedio, se obtiene lo siguiente cuando $y$ es positivo para el caso $i$ con un tratamiento $D$ positivo: $\operatorname{Avg}_{n}\left[y_{1 i} \mid D_{i}=1\right]$. Considerando el supuesto de efecto constante se puede advertir la existencia de un sesgo de selección que afecta a $\kappa$ y que consiste en la diferencia en el promedio de $y_{0 i}$ entre los grupos que son comparados:

$$
\begin{gathered}
\operatorname{Avg}_{n}\left[y_{1 i} \mid D_{i}=1\right]-\operatorname{Avg}_{n}\left[y_{0 i} \mid D_{i}=0\right] \\
=\left(\kappa+\operatorname{Avg}_{n}\left[y_{0 i} \mid D_{i}=1\right]\right)-\operatorname{Avg}_{n}\left[y_{0 i} \mid D_{i}=0\right] \\
=\kappa+\left(\operatorname{Avg}_{n}\left[y_{0 i} \mid D_{i}=1\right]-\operatorname{Avg}_{n}\left[y_{0 i} \mid D_{i}=0\right]\right)
\end{gathered}
$$

Es relevante considerar que $y$ no solo está determinada por $x$ o $D$. Si $y$ es la carrera política de $i$, es posible identificar otros determinantes. Por ejemplo, el capital familiar permite a $i$ tener ventajas debido a los procesos de cognición y socialización a los que estuvo expuesto. Además, desde un enfoque racional, $i$ recibe una transferencia de capital humano e incluso una ventaja de brand name que lo vuelve más competitivo para desarrollar una carrera política exitosa. En otras palabras, puede que $i$ sea un heredero de conexiones, redes y capital social, lo que aumenta sus probabilidades de desarrollar una carrera política ${ }^{18}$. ¿Cuál de todos estos factores es crítico? En este contexto, resulta complejo que en datos observacionales $D_{i}=1$ y $D_{i}=0$ se distribuyan de forma similar en los casos con diferentes resultados en la variable dependiente $y_{1 i}$ e $y_{0 i}$, lo que complejiza la selección aleatoria, bloqueando el camino metodológico más directo para eliminar el sesgo de selección. Probablemente un gran número de casos tenga capital familiar y sean exitosos, mientras que entre los casos no exitosos muy pocos tengan parentela política, por lo que se genera un desbalance.

En este sentido, resulta relevante eliminar el sesgo de selección que surge de diferencias no observadas. Por ejemplo, si se utiliza la siguiente ecuación:

$$
y_{i}=\alpha+\beta D_{i}+\gamma A_{i}+\epsilon_{i}
$$

dónde $\alpha$ es el equivalente a $\beta_{0}$, mientras $\beta$ y $\gamma$ equivalen a $\beta_{1}$ y $\beta_{2}$ en la notación

\footnotetext{
${ }^{18}$ Sobre capital familiar se recomienda revisar van Liefferinge y Steyvers (2009), Joignant (2014) y González-Bustamante (2014).
} 
clásica de mínimos cuadrados. Por otra parte, $D$ y $A$ son equivalente a $x_{1}$ y $x_{2}$, por tanto, su diferenciación es puramente conceptual y no formal. Si $A$, por ejemplo, equivale a una variable que mide pertenencia a un grupo, cuando hay más de dos grupos se necesitan $j-1$ variables dummy, es decir, $\sum_{j=1}^{n} \gamma_{j} A_{j i}$. En este ejemplo, $D$ podría ser el efecto de algún tipo de capital específico controlado por la membresía partidaria $A$ sobre la trayectoria $y$ del individuo $i$. Por último, $\epsilon_{i}$ representa los residuos, lo que no se aborda para no complejizar el ejemplo. Cuando $D_{i}$ es asignado aleatoriamente, la expectativa condicional de $y_{i}$ con $D_{i}=1$ es equivalente a cuando $D_{i}=0$, lo que puede ser complejo de obtener empíricamente con datos observacionales como se mencionó anteriormente:

$$
\mathbb{E}\left[y_{i} \mid D_{i}=1\right]=\mathbb{E}\left[y_{i} \mid D_{i}=0\right]
$$

Esta equivalencia permite una medición adecuada del efecto causal ya que se eliminan las diferencias sistemáticas entre grupos u observaciones como se aprecia a continuación:

$$
\begin{gathered}
\mathbb{E}\left[y_{i} \mid D_{i}=1\right]-\mathbb{E}\left[y_{i} \mid D_{i}=0\right] \\
=\mathbb{E}\left[y_{0 i}+\kappa \mid D_{i}=1\right]-\mathbb{E}\left[y_{0 i} \mid D_{i}=0\right] \\
=\kappa+\mathbb{E}\left[y_{0 i} \mid D_{i}=1\right]-\mathbb{E}\left[y_{0 i} \mid D_{i}=0\right] \\
=\kappa
\end{gathered}
$$

En consecuencia, si los datos no fueron asignados aleatoriamente, aunque la muestra sea probabilística, no se elimina el sesgo y, por tanto, las predicciones realizadas con modelos lineales o logísticos son imprecisas. Para solucionar esto existen dos caminos. El primer camino es más tradicional y se da cuando la posibilidad de selección aleatoria se encuentra bloqueada porque se trabaja con datos observacionales y no con un enfoque experimental, entonces para tratar de desbloquear la inferencia causal se puede recurrir a técnicas de emparejamiento o pareo que permitan generar agrupaciones similares con base en variables relacionadas con características y atributos de los casos. En este contexto, existen distintas técnicas para controlar el sesgo de omisión de variables y el sesgo de selección en el análisis de regresiones, basadas principalmente en emparejamiento e indicadores de propensión.

El segundo camino es menos ortodoxo y consiste en usar un enfoque basado en teoría de conjuntos y análisis comparado cualitativo (QCA, por sus siglas en inglés), sacrificando la lógica probabilística por una determinista. Sin embargo, al tomar este sendero surgen dos problemas. Primero, si bien QCA cuenta con extenso desarrollo 
que data de la década de los ochenta y de los trabajos de Charles C. Ragin ${ }^{19}$, no ofrece la confiabilidad que otorgan los modelos econométricos. Segundo, a pesar de que QCA es una técnica más bien mixta ya que si bien no sigue una lógica probabilística funciona con algebra booleana y teoría de conjuntos, algunos investigadores se aferran a la idea de que debe ser interpretado exclusivamente de forma cualitativa y solo usado en estudios con small- $N$. Esto ciertamente no es así y es posible encontrar un número considerable de trabajos que apelan a que incluso debe ser validado y complementando con regresiones.

\section{Reflexiones finales}

El estudio de los gobernantes es fascinante. Parafraseando a Mills, tratar de comprender por qué los individuos de los más altos círculos de poder no son representativos ya que su posición generalmente no está relacionada con sus capacidades, por el contrario, ha sido condicionada por las mismas fuentes poder y los mecanismos que lo delimitan, constituye un desafío intelectual relevante y ciertamente seguirá siéndolo mientras las sociedades se deban organizar políticamente.

En este contexto, es posible advertir ciertas dificultades teóricas. Por una parte, resulta complejo establecer un límite claro en el campo político entre las élites políticas y burocráticas. Por otro lado, la influencia del trabajo de Pierre Bourdieu también implica ciertas complejidades que se plasman, por ejemplo, en tipologías de políticos profesionales que pueden dificultar el análisis empírico en algunas oportunidades ya que resultan difusas y pueden asociarse a problemas de colinealidad en la medición.

De todas maneras, la investigación en la región ha sido prolífica centrándose, por una parte, en la profesionalización de la política, carreras y trayectorias, congruencia y composición social de los grupos elitarios y, por otra, en rotación y reclutamiento ministerial. En este artículo no se profundizó debidamente en la línea asociada al estudio de los altos directivos y los servicios civiles, sin embargo, es una línea interesante y creciente. También es posible identificar obras clave publicadas en los últimos años y la existencia de activos observatorios, redes y grupos de investigación.

Por último, si bien pueden existir diversas complejidades metodológicas al estudiar la élite política gubernamental, en este artículo se abordaron específicamente los problemas relacionados con la estrategia de identificación, la confiabilidad de la información, el análisis de vínculos entre actores y el riesgo de realizar comparaciones y predicciones sesgadas. Este último punto es particularmente delicado ya que aplicar modelos

\footnotetext{
${ }^{19}$ Véanse, por ejemplo, Ragin (1987, 2000, 2006).
} 
econométricos sin los controles apropiados puede, evidentemente, conducir a resultados espurios.

\section{Referencias}

Alcántara, M. (2012). El oficio de político. Tecnos, Madrid.

Alcántara, M. (2013). De políticos y política: profesionalización y calidad en el ejercicio público. Perfiles Latinoamericanos, 21(41):19-44.

Alcántara, M. y Luna, J. P. (2004). Ideología y competencia partidaria en dos posttransiciones: Chile y Uruguay en perspectiva comparada. Revista de Ciencia Política, $24(1): 128-168$.

Angrist, J. D. y Pischke, J.-S. (2015). Mastering Metrics. The Path from Cause to Effect. Princeton University Press, Nueva Jersey.

Bardi, L. y Pasquino, G. (1994). Euroministri. Il governo dell'Europa. Il Saggiatore, Milán.

Bobbio, N. (2003). Élites, teoría delle. En Bobbio, N., Matteucci, N., y Pasquino, G., editores, Dizionario di Politica. Unione Tipografico-Editrice Torinese, Turín.

Bourdieu, P. (2009/1980). El sentido práctico. Siglo XXI Editores, Ciudad de México.

Buquet, D. y Selios, L. (2017). Political Congruence in Uruguay, 2014. En Joignant, A., Morales, M., y Fuentes, C., editores, Malaise in Representation in Latin American Countries. Chile, Argentina, and Uruguay. Palgrave Macmillan, Nueva York.

Camerlo, M. y Martínez-Gallardo, C. (2018). Government Formation and Minister Turnover in Presidential Cabinets: Comparative Analysis in the Americas. Routledge, Nueva York.

Camerlo, M. y Pérez-Liñán, A. (2015). Minister Turnover, Critical Events, and the Electoral Calendar in Presidential Democracies. The Journal of Politics, 77(3):608619.

Castellani, A. G. y Dulitzky, A. (2018). The Reverse Revolving Door: Participation of Economic Elites in the Public Sector during the 1990s in Argentina. Latin American Business Review, 19(2):131-156. 
Cisternas, C. y González-Bustamante, B. (2016). Los monjes de CIEPLAN: Producción intelectual y redes de citación durante los años ochenta. En Seminario Internacional Ciencias sociales en la encrucijada: Intelectuales y tecnócratas latinoamericanos en tiempos de autoritarismo (1969-1990), Santiago. Universidad Bernardo O'higgins.

Cisternas, C. y Vásquez, J. (2018). Comisiones Asesoras Presidenciales en Chile: Entre la expertise y la pluralidad de actores sociales. European Review of Latin American and Caribbean Studies, (106):1-24.

Codato, A. (2015). Metodologias para a identificação de elites: três exemplos clássicos. En Perissinotto, R. y Codato, A., editores, Como estudar elites. Editora Universidade Federal do Paraná, Curitiba.

Codato, A. y Espinoza, F. (2018). Las élites en las Américas: Diferentes perspectivas. Editora Universidade Federal do Paraná, Curitiba.

Codato, A., Perissinotto, R., Dantas, E. G., y Cavalieri, M. (2019). La connexion financière. La porte tournante de la Banque centrale du Brésil. Gouvernement et action publique, 8(1):137-155.

Cuevas, H. (2015). Elites política y trayectorias políticas militantes: El espacio sociopolítico de la izquierda chilena. Revista de Ciencia Política, 35(2):299-325.

Cuevas, H., Morales, M., Rojas, J., y Aubry, M. (2015). Los ministros de la Concertación de Partidos por la Democracia en Chile. Un análisis de sus capitales político, social y cultural. América Latina Hoy, (71):121-150.

Delamaza, G. (2013). De la élite civil a la élite política. Reproducción del poder en contextos de democratización. Polis, 12(36):299-325.

Dowding, K. y Dumont, P. (2015). The Selection of Ministers Around the World. Routledge, Nueva York.

Espinoza, V. (2010). Redes de poder y sociabilidad en la élite política chilena. Los parlamentarios 1990-2005. Polis, 9(26):251-286.

Garrido-Vergara, L. (2020). Political, Social, and Cultural Capital in the Chilean Political Elite, 1990-2010. Latin American Politics and Society, 62(1):121-141.

González-Bustamante, B. (2013a). Factores de acceso y permanencia de la élite política gubernamental en Chile (1990-2010). Política, Revista de Ciencia Política, 51(1):119153. 
González-Bustamante, B. (2013b). Trayectorias y patrones de carreras políticas de ministros en Chile (1990-2010). En XI Congreso nacional de Ciencia Política, Paraná. Sociedad Argentina de Análisis Político.

González-Bustamante, B. (2014). Elección directa de consejeros regionales 2013. Rendimiento del capital político, familiar y económico en una nueva arena electoral en Chile. Política, Revista de Ciencia Política, 52(2):49-91.

González-Bustamante, B. (2019a). Brechas, representación y congruencia éliteciudadanía en Chile y Uruguay. Convergencia Revista de Ciencias Sociales, (80):1-27.

González-Bustamante, B. (2019b). The Politics-Administration Dichotomy: A Case Study of the Chilean Executive during the Democratic Post-Transition. Bulletin of Latin American Research. Early View.

González-Bustamante, B. y Cisternas, C. (2016). Élites políticas en el poder legislativo chileno: la Cámara de Diputados (1990-2014). Política, Revista de Ciencia Política, $54(1): 19-52$.

González-Bustamante, B. y Cisternas, C. (2020). Aplicación de ForceAtlas2, un algoritmo de diseño gráfico continúo, para el estudio de las élites. Tufte Working Papers, (1):1-15.

González-Bustamante, B. y Garrido-Vergara, L. (2018). Socialización, trayectorias y poscarrera de ministros en Chile, 1990-2010. Política y Gobierno, XXV(1):31-64.

González-Bustamante, B. y Olivares, A. (2015). Rotación de subsecretarios en Chile. Una exploración de la segunda línea gubernamental (1990-2014). Revista de Gestión Pública, IV(2):151-190.

González-Bustamante, B. y Olivares, A. (2016). Cambios de gabinete y supervivencia de los ministros en Chile durante los gobiernos de la Concertación (1990-2010). Colombia Internacional, (87):81-108.

González-Bustamante, B. y Olivares, A. (2018). La élite política gubernamental en Chile: Supervivencia de ministros. En Codato, A. y Espinoza, F., editores, Las élites en las Américas: Diferentes perspectivas. Editora Universidade Federal do Paraná, Curitiba.

Hanneman, R. A. y Riddle, M. (2005). Introduction to Social Networks Methods. University of California Riverside, Riverside. 
Imai, K. (2017). Quantitative Social Science: An Introduction. Princeton University Press, Nueva Jersey.

Joignant, A. (2011a). Tecnócratas, technopols y dirigentes de partido: tipos de agentes y especies de capital en las elites gubernamentales de la Concertación (1990-2010). En Joignant, A. y Güell, P., editores, Notables, tecnócratas y mandarines: Elementos de sociología de las elites en Chile (1990-2010). Ediciones Universidad Diego Portales, Santiago.

Joignant, A. (2011b). The Politics of Technopols: Resources, Political Competence and Collective Leadership in Chile, 1990-2010. Journal of Latin American Studies, $43(3): 517-546$.

Joignant, A. (2012). Habitus, campo y capital. Elementos para una teoría general del capital político. Revista Mexicana de Sociología, 74(4):587-618.

Joignant, A. (2014). El capital político familiar: Ventajas de parentela y concentraciones de mercado en las elecciones generales chilenas 2013. Política, Revista de Ciencia Política, 52(2):13-48.

Joignant, A. y Güell, P. (2011). Notables, tecnócratas y mandarines: Elementos de sociología de las elites en Chile (1990-2010). Ediciones Universidad Diego Portales, Santiago.

Joignant, A. y González-Bustamante, B. (2014). El núcleo de la élite política chilena 1990-2010. Proyecto Fondecyt 1100877. Working Paper, Universidad Diego Portales.

Joignant, A., Perelló, L., y Torres, J. (2012). Las fuentes del poder político. Fundamentos para una teoría del capital político a partir de la evidencia chilena. En XXII Congreso Mundial de Ciencia Política, Madrid. International Political Science Association.

Joignant, A., Perelló, L., y Torres, J. (2015). Political Capital and the Unequal Career Origins of the Political Elite in Chile. En Political Inequality in an Age of Democracy. Cross-National Perspectives. Routledge, Nueva York.

Kerby, M. (2015). Canada: Ministerial Careers. En Dowding, K. y Dumont, P., editores, The Selection of Ministers Around the World. Routledge, Nueva York.

Lorrain, F. y White, H. C. (2012/2003). La equivalencia estructural de losindividuos en las redes sociales. En Requena Santos, F., editor, Análisis de redessociales. Orígenes, teorías y aplicaciones. Centro de Investigaciones Sociológicas, Madrid. 
Luna, J. P. y Zechmeister, E. J. (2005). Political Representation in Latin America: A Study of Elite-Mass Congruence in Nine Countries. Comparative Political Studies, 38(4):388-416.

Lupu, N. y Warner, Z. (2017). Mass-Elite Congruence and Representation in Argentina. En Joignant, A., Morales, M., y Fuentes, C., editores, Malaise in Representation in Latin American Countries. Chile, Argentina, and Uruguay. Palgrave Macmillan, Nueva York.

Maillet, A., González-Bustamante, B., y Olivares, A. (2016). ¿Puerta giratoria? Análisis de la circulación público-privada en Chile (2000-2014). Working Paper 7, Programa de las Naciones Unidas para el Desarrollo.

Maillet, A., González-Bustamante, B., y Olivares, A. (2019). Public-Private Circulation and the Revolving Door in the Chilean Executive Branch (2000-2014). Latin American Business Review, 20(4):367-387.

Martínez-Gallardo, C. (2014). Designing Cabinet: Presidential Politics and Ministerial Instability. Journal of Politics in Latin America, 6(2):3-38.

Mills, C. W. (2013/1956). La élite de poder. Fondo de Cultura Económica, Ciudad de México.

Morales, J. J. (2016). Entre la ciencia y la política: la forja de una élite intelectual latinoamericana. Política, Revista de Ciencia Política, 54(2):157-188.

Olivares, A., González-Bustamante, B., Toro, S., Arellano, J. C., Yanes-Rojas, A., Zurita-Tapia, J., Lopes, A. V., Robelo Guzmán, C., y Canavesi Sosa, J. B. (2020). Nuevos desafíos, enfoques y perspectivas para estudiar élites políticas. Iberoamericana, XX(74):229-259.

Pasquino, G. (2000). La clase política. Acento, Madrid.

Pasquino, G. (2011). Nuevo curso de ciencia política. Fondo de Cultura Económica, Ciudad de México.

Perissinotto, R. y Codato, A. (2015). Como estudar elites. Editora Universidade Federal do Paraná, Curitiba.

Peters, B. G. (1995). The Politics of Bureaucracy. Longman, Nueva York. 
Putnam, R. D. (1976). The Comparative Study of Political Elites. Prentice Hall, Nueva Jersey.

Quiroz Flores, A. (2015). United States of America: The Cabinet. En Dowding, K. y Dumont, P., editores, The Selection of Ministers Around the World. Routledge, Nueva York.

Ragin, C. C. (1987). The Comparative Method: Moving Beyond Qualitative and Quantitative Strategies. University of California Press, Berkeley.

Ragin, C. C. (2000). Fuzzy-Set Social Science. University of Chicago Press, Chicago.

Ragin, C. C. (2006). Set Relations in Social Research: Evaluating Their Consistency and Coverage. Political Analysis, 14(3):291-310.

Rovira, C. (2011). Hacia una sociología histórica sobre las elites en América Latina: un diálogo crítico con la teoría de Pierre Bourdieu. En Joignant, A. y Güell, P., editores, Notables, tecnócratas y mandarines: Elementos de sociología de las elites enChile (1990-2010). Ediciones Universidad Diego Portales, Santiago.

Silva, P. (2009). In the Name of Reason: Technocrats and Politics in Chile. Penn State University Press, University Park.

Urizzi Cervi, E. (2015). Análise de elites em perspectiva relacional: a operacionalizaçãoda Análise de Redes Sociais (ARS). En Perissinotto, R. y Codato, A., editores, Como estudar elites. Editora Universidade Federal do Paraná, Curitiba.

van Liefferinge, H. y Steyvers, K. (2009). Family matters? Degrees of family politicization in political recruitment and career start of mayors in Belgium. Acta Politica, 44(2):125-149.

Vommaro, G. y Gené, M. (2018). Las élites políticas en el Sur. Un estado de la cuestión de los estudios sobre la Argentina, Brasil y Chile. Ediciones Universidad Nacional de General Sarmiento, Munro.

Waldo, D. (1955). The Study of Public Administration. Doubleday and Company, Garden City.

Wasserman, S. y Faust, K. (1994). Social Network Analysis: Methods and Applications. Cambridge University Press, Nueva York.

Weber, M. (1978/1922). Economy and Society. University of California Press, Berkeley. 
Weber, M. (2015/1919). Politics as Vocation. En Waters, T. y Waters, D., editores, Weber's Rationalism and Modern Society. Palgrave Macmillan, Nueva York.

Wilson, W. (1887). The Study of Administration. Political Science Quarterly, 2(2):197222.

\section{Apéndice}

\section{Historial de revisiones}

\section{Revisión Fecha Descripción}

1,0 29 feb, 2020 Manuscrito original (MO) enviado a revisión

$2,0^{\star} \quad 11 \mathrm{abr}, 2020 \quad$ Manuscrito aceptado (MA)

$3,0^{\star} \quad 16$ abr, $2020 \quad$ MA revisado

$4,0^{\star} \quad 22$ may, $2020 \quad$ MA revisado

$5,0^{\star} \quad 23$ jun, $2020 \quad$ MA revisado

$6,0^{\star} \quad 15 \mathrm{sep}, 2020 \quad$ Versión de registro (VDR)

$7,0^{\star} \quad 25 \mathrm{sep}, 2020 \quad$ VDR revisada

$8,0^{\star} \quad 03$ nov, 2020 VDR revisada

屯 Descargar la versión más reciente desde SocArXiv (DOI: 10.31235/osf.io/syqu4).

セ Descargar la versión publicada (DOI: 10.18441/ibam.20.2020.74.229-259).

* versiones disponibles en línea. 